\title{
A Survey of Routing Protocols for Energy Constrained Ad Hoc Wireless Networks
}

\author{
Natalia Vassileva, Francisco Barcelo-Arroyo \\ Department of Telematic Engineering \\ Technical University of Catalonia (UPC) \\ natalia@entel.upc.edu,barcelo@entel.upc.edu
}

\begin{abstract}
In this survey we review energy-aware routing protocols for wireless multihop ad hoc networks and critically discuss the main results in this area. The classification presented is in no case unique but summarizes the chief characteristics of the many published proposals for energy conservation. A common pitfall detected in most of the studies is the lack of unambiguous notion of network's lifetime and hence of clear objective of the designed algorithm. We, therefore, define first what operational lifetime for ad hoc networks means and then analyze the achievements from that angle. After getting insight into the different energy-aware routing protocols we point out another approach for extending network's operational lifespan, which has been overlooked in the relevant literature.
\end{abstract}

\section{Introduction}

One of the paramount deficiencies of wireless mobile multihop ad hoc networks when the constituent nodes are battery supplied is that they are limited in energy which directly affects the connectivity of the network and its lifetime. Since the majority of the provisioned applications are energy-dependent this problem has gained popularity in the research community and the published work addressing it is immense. This survey comprises the routing part of the proposals and is confined to the fundamental principles and generic techniques used for efficient utilization of the resources in ad hoc networks on the network layer.

We elaborated the essence of the problem which guided our understanding and led to a comprehensive definition of operational lifetime for MANETs, something we discuss in the first part of this work. Then we present a concise review of the routing protocols with energy metrics which aim at postponing the time until network partitioning. The next part of the survey is focused on load balancing in ad hoc networks since it has immediate effect on the consumption of energy although never investigated from that perspective.

The consulted literature shows that load balancing strategies for ad hoc networks have been explored in the same context as in the wired networks - prevention and/or alleviation of congestion and fault tolerance (note that in the wired world the problem of limited energy is not present). However, the equitable use of the network's resources leads to more efficient energy use which is essential for ad hoc networks. Hence, the second taxonomy of load balancing protocols is as extensive as the first part of our study.

The protocols revised are thought for generalpurpose mobile ad hoc networks. Sensor networks are out of the scope of this work.

\section{Problem Statement}

In contrast to the current cellular networks, which rely on a wired structure with wireless last hop, ad hoc wireless networks are infrastructureless. The nodes which comprise them have routing capabilities and forward traffic for other communicating parties that are not within the transmission range of each other. They are characterized with lower computing and energy resources. Ad hoc routing as a result is challenged by power and bandwidth constrains as well as frequent topology changes, which it must adapt to and converge quickly. Conventional routing protocols for wired networks can not be employed in such an environment by the virtue of the factors enumerated. This fact has motivated the design of ad hoc-specific routing protocols, which initially have had as a main criterion minimum number of hops. The prime deficiency of this criterion is that selecting the routes obeying the min-hop principle does not protect nodes from being overused. If the nodes are not equitably loaded with networking activities those of them, which are preferably used (to forward other nodes' traffic) will 
exhaust their energy reserves faster. Since these nodes usually lie on many paths, when they run out of power the network will become partitioned and consequently some sessions will be disconnected.

The lifetime of the network in the scientific papers reviewed is usually defined according to the following criteria: 1) the time period until the first node burns out its entire battery budget, 2) the time until certain percentage of the nodes fail, 3) the time until network partitioning.

The problem of node's failure, and as a result network partitioning, is serious in ad hoc networks (in contrast to sensor networks, where as pointed out in [3], the single sensor failure is usually unimportant if it does not lead to loss of sensing and communication coverage). Ad hoc networks are oriented towards personal communications and the loss of connectivity to any node is of significance. Here, as a case of example, we consider a disaster recovery event but argue that the same is true for most of the applications ad hoc networks are envisioned for. In such scenarios, it is of main importance that fire-fighters or other rescuers have connectivity with all of the members (maintain the connectivity between all the constituent nodes) on the one hand and on the other each member should have connectivity with the rest of the group (the network should be preserved in its entirety for the duration of the rescue operation).

When energy metrics are used for the design of the routing protocols the ultimate goal is to maintain the network connected and extend the time until it gets partitioned. The conservation or the efficient usage of the battery budget of the nodes is a way to achieve this rather than an objective.

Network partitioning causes interruption of the communication sessions and can be invoked by node movement or by node failure due to energy expenditure. Whereas the former can not be controlled by the routing protocol, the later one can be avoided through appropriate routing decisions. Operational lifetime is therefore defined in this survey as the time until network partitioning due to battery outage.

In order to achieve these objectives (maintaining connectivity and for as long as possible), the distribution of the network's tasks between all its nodes should be equal so that all of them decrease their power savings at the same rate and eventually run out of energy at approximately the same time. From the design perspective, it is much more demanding to achieve simultaneous failure of the nodes (due to lack of energy), so that personal (no node powers down before the others) and network requirements (no partitioning) are met. Network operational lifetime, therefore, concerns the relative and not absolute lifetime of the network. It is measured as a time period until all nodes simultaneously run out of energy and compared to the lifetime of a network which is not energy-aware (min-hop-wise e.g.) can have smaller absolute value. From engineering and application perspective however, the former time span is much more interesting and meaningful (consider a case when some nodes have their batteries full but because they belong to disconnected parts of the network or have to communicate with nodes that are turned down due to energy deficiency, can not succeed in communicating; they will add to the absolute lifetime of the network but this is not of practical interest). It should be clarified that by equal loading of the nodes we mean engaging them in network activities in accordance to their relative energy savings (the ratio of the current battery capacity to its full state), since the network can be heterogeneous in respect to the battery capacities of its constituent nodes.

The research work dealing with the formulated problem is vast, but most studies share common principles. The literature review, therefore, summarizes the basic ideas rather than covering all protocols and their modifications and extensions.

\section{Energy-Aware Routing}

\subsection{Residual Energy}

The fundamental idea of energy-aware routing is to consider as a prime metric for the selection of a route the energy status of the nodes. The diversity of the approaches, which use the residual energy (the general form of the function applied for calculating it is $\left.f_{i}\left(c_{i}^{t}\right)=\frac{1}{c_{i}^{t}},[22]\right)$, is manifested in the design of the cost function, viz. all have the same metric although approximated by different functions. Toh et al. in [22] e.g. propose a Min-Max Battery Capacity Routing (MMBC) to exclude the energy starving nodes (those having least battery capacity) from route selection. The idea of [20] to use shortest hop routing until the nodes consume a predefined part of their energy and then switch to an algorithm with power-aware metrics has been adopted and elaborated in [21]. The behaviour of a Conditional MMBCR (CMMBCR) routing algorithm is in function of the battery capacity of the nodes.

\subsection{Energy Drain Rate}

The energy drain rate ([8]), introduced by Kim et al., represents the speed of energy consumption and has an added value for network provisioning and monitoring. Knowing the lifetime of the node, the traffic passing through it can be deviated when needed 
in order to avoid node's failure due to battery outage. In the work of Kim et al. [11] the new routing metric proposed for predicting battery lifetime, i.e. the energy drain rate, is computed by exponential weighted moving average method and gives the estimated energy dissipation per second. The cost function is defined as the ratio between the residual battery power $(R B P)$ and drain rate $(D R)$ at a node: $C=\frac{R B P}{D R}$. The Minimum Drain Rate (MDR) mechanism is a max-min algorithm (an analogue to MMBCR) which implements the above cost function. Kim et al. in [9] extend the MDR algorithm to Conditional MDR, which shares the same objective and principles with CMMBR.

\subsection{Local Routing}

In the generic on-demand ad hoc algorithms (denoted as global in the work of Woo et al., [28]) all nodes participate in the phase of path searching, while the final decision is made in the source or destination node. Woo et al. [28] grant the permission for deciding to participate into route searching to each node in the network, this way distributing the decision-making process to all nodes. Local Energy-Aware Routing (LEAR) algorithm has as a prime criterion the energy profile of the nodes which defines the reluctance/willingness of intermediate nodes to respond to route requests and forward data traffic. The technique of shifting the responsibility for reacting to changes in the energy budget of the nodes from the source-destination nodes to the intermediate leads to avoiding the need for periodically exchanging control information. It is commonly used for improving the performance of the routing protocols in many later approaches. This mechanism is inventive but depends on the way it is implemented (in [6] e.g., the proposed SEADSR protocol is bias towards highly powered routes: it does not execute a monitoring function and a lot of traffic can be forwarded by nodes which at the time the route was selected were highly powered).

\subsection{Expected Energy Consumption}

The work presented in [13] is designed from extending operational lifetime perspective and is founded on the expected energy consumption idea. Misra and Banerjee, [13] propose and evaluate the performance of Maximum Residual Packet Capacity (MRPC) algorithm which uses residual battery as a factor in route selection decision but also add the expected energy spent metric, which is the main contribution. The cost metric thus comprises node- specific parameter - battery power - and link-specific parameter - packet transmission energy - for reliable communication across the link. The main motivation is that link characteristics can significantly affect the energy consumption and can lead to excessive retransmissions of packets. The cost of choosing a particular link is defined as the maximum number of packets that can be transmitted by the transmitting node over the specific link, assuming the complete absence of any other cross traffic at that node [13]. The maximum lifetime of a given path is determined by the weakest intermediate node (the one with smallest cost).

\subsection{Battery-Sensitive Routing}

A completely different approach is presented in [5] by Chiasserini and Rao, and consequently by Ma and Yang [30]. The algorithms proposed by them make use of the available battery capacity by battery-sensitive routing. Both works [5 and 30] study the lifetime of the battery and based on the two processes: recovery (reimbursement) and discharging loss (over-consumed power), experienced when no/new traffic is transmitted, design a cost function which value reflects the energy consumption. The selection function in [5] is a minimum function over the cost functions of all routes. The sensitivity of the algorithm in [30] towards battery behaviour makes it switch between different paths so that battery recovery can take place and the maximum of the node's battery capacity can be attained.

\subsection{Common Deficiencies}

A common characteristic of the previously cited studies is the fact that a min-max cost function is applied. The min-max algorithms are implemented in order to overcome the problem introduced when the total energy cost of routes is used as argument for the selection of a route, i.e. to exclude nodes with small energy reserves. However, if these protocols are analyzed from network's operational lifetime perspective the problem to extend the network's lifespan for as long as possible persists. The deviation of the energy of the nodes along the path, as can be observed from the simulation results presented in [4] (the most recent and comprehensive study to evaluate the performance of some of the energy aware routing protocols), demonstrates that the distribution of the energy is not even in neither protocol, i.e. a particular view (the energy state of the nodes) without the global one (the distribution of the energy along the routes) does not conduct to an optimal result when considering the operational lifetime of the network. 
The protocols denominated as energy-aware usually take into account only energy-wise metrics and no other parameters. An improvement on this general approach is the inclusion of the speed with which the battery is burned. The energy drain rate is helpful to salvage a node from powering down by deviating traffic when a certain threshold is reached. However, if no additional metric is considered (as e.g. the load), it is an omission of the principle characteristics of the system, such as the fact that neighbouring nodes, when engaged in transmitting packets, are competing for the wireless medium (a fact pointed out in [18] apart from other MAC related studies). The consequent collisions and retransmissions are energy consuming and can not be represented by the residual energy metric. As discussed in the section that follows, the load at each node and in its neighbouring nodes is an indicator for the energy to be consumed for transmitting packets by the node in question. Moreover, it accounts for the shared nature of the radio medium. The network tasks with which each node is entrusted are a main article in the battery budget and when considered along with the current energy state of a node can regulate the speed with which the energy is consumed.

Although the results in the consulted scientific papers always show an improvement of the energy balance they are not representative enough not just because they are usually compared with proposals that do not contemplate the energy metric but also due to the fact that because of the design of the cost functions implemented can not achieve an equilibrium of the network activities between the constituent nodes.

\section{Load Balancing}

Load balancing techniques play an important role in achieving good network performance through a better spreading of traffic flows. They can alleviate and even prevent the effects of congestion, such as longer packet latency, poor packet delivery (throughput), high routing overhead. In MANETs, in contrast to wired networks, due to the bandwidth and power limitations, the consequences of traffic congestion are further worsen - excessive consumption of network resources: fast battery depletion of the most congested nodes and consequent partitioning of the network.

As pointed out earlier load balancing in ad hoc networks is developed from the same angle as in wired networks. However, the cited works are discussed here from the energy perspective.

\subsection{Single Path Routing}

Single path routing makes use of the best path according to some criterion, although during the route discovery procedure more than one prominent route could be found.

A common characteristic of most of the single path load balancing protocols (as e.g. [23, 24 and 31]) is the application of a suppression or a prevention policies. Both of these treat intermediate nodes in the same manner - intermediate nodes do not respond to route requests. The objective of the suppression policy is to avoid using the most loaded nodes and prevention is used in order to obtain fresh routing information. Although not implemented for energy reasons, the former one influences the energy status of the nodes and can be applied for energy conservation.

The distinguishing feature of LBAR algorithm [7], latter used in [14], is the introduction of the traffic load at neighbouring nodes (denoted as "traffic interference" in [7] and as "contention node" in [14]). Traffic interference accounts for the possible radio interference due to traffic load and manifests the shared nature of the wireless medium, which as clarified in the previous section is of importance for achieving energy efficiency. Wu and Harms in [26] present a mathematical approach which utilizes statistical information (standard deviation of the path load e.g.) in order to evenly distribute the load.

The main drawback in the context of energy conservation of the protocols consulted is that the total and average values of the observed parameters are not enough to achieve even allocation of the traffic load. If only the average values of the load metrics are relayed then there will not be a clear indicator whether some of the nodes along the path are heavily loaded or not, viz. no clear reference for the distribution of the load along the path. The work of Hassein and Zhou [7] does consider the traffic load at neighbouring nodes besides the load at the node, but for the best route to be selected the algorithm compares the minimum traffic in transmission and minimum interference - it can guarantee that heavily loaded nodes are excluded but does not fulfill the requirement for even distribution of the traffic load. Only Wu and Harms in [26] take into consideration the standard deviation apart from the average load in its comparison function.

\subsection{Multipath Routing}

The trends in using multipath techniques for routing in ad hoc networks are subject to different purposes pursued. Initially, the multipath routing was proposed in the light of providing fault tolerance [14, 12]. In [1] it is applied to guarantee differentiated treatment to priority traffic and in [2] as a means for minimizing the 
overhead, resulting from route disruptions and consequent flooding of route requests. Wang et al. [27], Zhang et al [31], and Yin and Lin [29] contemplate multipath routing from load balancing perspective.

Multipath routing is regarded as appealing for ad hoc networking because it can provide fault tolerance. The use of back-up routes leads to less packet loss, can ensure longer duration of the communication session and robustness to mobility and fading. Moreover, dispatching the data packets of each flow through many network nodes along different paths can lead to better distribution of the traffic load as demonstrated in the study of Parissidis et al. [16] and as a consequence to more even distribution of the residual energy.

Although multipath routing can positively influence the energy consumption in the network there are some open issues to be considered and successfully addressed for the load balancing to be implemented in the ad hoc routing protocols. One of them is the possible augmentation of the total overhead and packet disorder, which negatively influences some services (as e. g. those working over TCP) $[2,25]$. The nature of the shared radio medium impacts the proper work of multipath technique as well, since the paths should be node and link-disjoint [15], which makes the mechanisms employed much more complex in comparison to the single path routing. Another question is whether route maintenance should be centralized or distributed [2, 14 and 17].

\subsection{Common Deficiencies of Load Balancing Routing Protocols in the Context of Extending Operational Lifetime}

Some of the prime limitations of the load balancing protocols from energy efficiency perspective are next specified.

Although the main objective of the load balancing routing is efficient utilization of network resources, none of the studies revised above takes into account energy-wise metrics. Doubtless that better distribution of load leads to more efficient use of bandwidth which means less contention to be observed and consequently less energy consumed, it is not self-contained for achieving complete energy efficiency. Ad hoc networks are not necessarily energy-homogeneous, so that the information of the nodes' load tasks to be enough for energy-wise selection of the paths. Current load of a node can approximate the future dissipation of the energy but does not contain the history of the past activities and leaves the residual energy level of the node hidden.
Two studies investigate the idea of including energy and load balancing metrics in routing algorithm in order to prolong network's lifetime. In [19] the authors point out that the queue length when taken as a parameter of the routing protocol has a direct impact on the distribution of the traffic flows and in particular on the energy consumption. Nonetheless, the idea discussed in [19] is not elaborated further. A recent study [10], explores for the first time the energy and load metrics as part of the path search of the routing algorithm. Kim et al., [10] consider nodes' traffic load and lifetime deviation along with their energy status to prolong network's lifetime and achieve load balancing (although we argue that the latter one is a mechanism rather than an objective). They use the criterion introduced first by Kim et al. in [8] that reflects node's lifetime. The energy dissipation rate gives the estimated energy consumption per unit time. Route's lifetime is used as a route selection criterion - the route with the longest predicted lifetime is selected for forwarding traffic flows. The algorithm forms part of max-min algorithms - in determining path lifetime, the minimum lifetime of the intermediate nodes is used. The proposal is the first to observe the interaction between the discussed routing metrics. One of its deficiencies is that it does not overcome the weakness of MMBCR approach: similar to its predecessor, the lifetime of the route is determined by the node with the shortest lifetime among all the intermediate nodes. This way the nodes with the lowest remaining energy are avoided, but the scheme does not guarantee an even distribution of the load along the network. Another pitfall is the lack of route monitoring function, which is essential for achieving energy efficiency as mentioned earlier.

\section{Conclusions}

The consulted studies, although aiming at energy conservation and better utilization of network's resources consider some but not all aspects of the problem. Consequently, the solutions proposed attack restricted number of factors and do not observe the whole palette of metrics that influence the final performance of the network.

Energy-constrained routing and load-balance routing are mutually related and employing one of them leads to improving the other. However, the synergy of the revised techniques is not investigated. The notion of energy-aware routing should be enriched with load-balancing techniques.

\section{Acknowledgments}


This research was funded by the Spanish Government and FEDER through the Plan Nacional de I+D (TEC2006-09466/TCM).

\section{References}

[1] G. Alandjani, E. E. Johnson, "Fuzzy routing in ad hoc networks", in Proc. IEEE $22^{\text {nd }}$ IPCCC, 2003, pp. 525- 530.

[2] N. Akhtar, R. Tafazolli, "Traffic-based multipath routing for mobile ad hoc networks", in Proc. IEEE Int. Workshop on Wireless Ad-Hoc Networks, 2004, pp. 201 - 206.

[3] S. Basagni, M. Conti, S. Giordano, Iv. Stojmenovic, "Chapter 11: Energy-efficient communication in ad hoc wireless networks", Mobile ad hoc networking, Wiley-IEEE Press, 2004.

[4] L. Cao, T. Dahlberg, Y. Wang, "Performance evaluation of energy efficient ad hoc routing protocols", in Proc. IEEE IPCCC, 2007, pp. $306-313$.

[5] C.-F. Chiasserini and R. R: Rao, "Routing protocols to maximize battery efficiency", in Proc. IEEE MILCOM, 2000, pp. $496-500$.

[6] M. C. Domingo, D. Remondo, O. Leon, “A simpler routing scheme for improving ad hoc network survivability", in Proc. IEEE GLOBECOM, 2003, pp.718 - 723.

[7] H. Hassanein, A. Zhou, "Routing with load balancing in wireless ad hoc networks", in Proc. $4^{\text {th }}$ MSWiM, 2001, pp. 89 $-96$.

[8] D. Kim, G. - L. Aceves, K. Obraczka, J -C. Cano, P. Manzoni, "Power-aware routing based on the energy drain rate for mobile ad hoc networks", in Proc. $14^{\text {th }}$ ICCCN, 2002 , pp. $565-569$.

[9] D. Kim, G. - L. Aceves, K. Obraczka, J -C. Cano, P. Manzoni, "Routing mechanisms for mobile ad hoc networks based on the energy drain rate", in IEEE Trans. Mobile Computing, April - June 2003, vol. 2, no. 2, pp. 161 - 173.

[10] D.-H. Kim, R. Ha, H. Cha, "Traffic load and lifetime deviation based power-aware routing protocol for wireless ad hoc networks", in Proc. $4^{\text {th }}$ WWIC, 2006, pp. $325-336$.

[11] B. C. Kim, J. Y. Lee, H. S. Lee, J. S. Ma, "An ad-hoc routing with minimum contention time and load balancing", in Proc. IEEE GLOBECOM, 2003, pp. $81-85$.

[12] S. J. Lee, M. Gerla, “AODV-BR: backup routing in ad hoc networks", Proc. IEEE WCNC, 2000, pp. $1311-1316$.

[13] A. Misra and S. Banerjee, "MRPC: Maximizing network lifetime for reliable routing in wireless environments", in Proc. IEEE WCNC, 2002, pp. $800-806$.

[14] A. Nasipuri, S. R. Das, "On-demand multipath routing for mobile ad hoc networks", in Proc. IEEE $8^{\text {th }}$ ICCCN, 1999, pp. $64-70$.

[15] M. R. Pearlman, Z. J. Haas, P. Sholander, S. S. Tabrizi, "On the impact of alternate path routing for load balancing in mobile ad hoc networks", in Proc. 1st ACM MobiHoc, 2000, pp. $3-10$.

[16] G.Parissidis, V. Lenders, M. May, B. Plattner, "Multipath routing protocols in wireless mobile ad hoc networks: a quantitative comparison", in Proc. 6th NEW2AN, 2006, pp. $313-326$.

[17] P. P. Pham and S. Perreau, "Performance analysis of reactive shortest path and multi-path routing mechanism with load balance", in Proc. $22^{\text {nd }}$ INFOCOM, 2003, pp. 251 - 259. [18] A. Safwat, H. Hassanein, H. Mouftah, "Energy-aware routing in MANETs: analysis and enhancements", in Proc. 5th ACM/IEEE Int. MSWiM, 2002, 46 - 53.

[19] L. M. Sánchez, N. V. Vassileva, F. Barceló, P. F. Carbajo, "Energy and delay-constrained routing in mobile ad hoc networks: an initial approach" in Proc. ACM Int. Workshop PE-WASUN, 2005, pp. $262-263$.

[20] S. Singh, M. Woo, C. S. Raghavendra, "Power-aware routing in mobile ad hoc networks", in Proc. $4^{\text {th }}$ ACM/IEEE MobiCom, 1998, pp. $181-190$.

[21] C. - K. Toh, "Maximum battery life routing to support ubiquitous mobile computing in wireless ad hoc networks", IEEE Comm. Mag., June 2001, vol.39, issue6, pp. 138 - 147.

[22] C. - K. Toh, H. Cobb, D. A. Scott, "Performance evaluation of battery-life-aware routing schemes for wireless ad hoc networks", in Proc. IEEE ICC 2001, pp. 2824 - 2829.

[32] S-J. Lee, M. Gerla, "Dynamic load-aware routing in ad hoc networks", in Proc. IEEE ICC, 2001, pp. 3206 - 3210. [31] Y. J. Lee, G. F. Riley, “A workload-based adaptive loadbalancing technique for mobile ad hoc networks", in Proc. IEEE WCNC, 2005, pp. $2002-2007$.

[24] A. Valera, W. K. G. Seah, S. V. Rao, "Cooperative packet caching and shortest multipath routing in mobile ad hoc networks", in Proc. 22 $2^{\text {nd }}$ Annual Joint Conf. IEEE INFOCOM, 2003, pp. $260-269$.

[25] K. Wu, J. Harms, "Load-sensitive routing for mobile ad hoc networks", in Proc. IEEE $10^{\text {th }}$ ICCCN, 2001, 540 - 546.

[26] L. Wang, L. Zhang, Y. Shu, M. Dong, "Multipath source routing in wireless ad hoc networks", in Proc. IEEE Canadian Conf. Electrical and Computer Engineering, 2000, pp. $471-483$.

[27] K. Woo, C. Yu, D. Lee, H. Youn, B. Lee, "Nonblocking, localized routing algorithm for balanced energy consumption in mobile ad hoc networks", in Proc. ACM/IEEE MSCOTS, 2001, pp.117 - 124.

[28] S. Yin, X. Lin, "Multipath minimum energy routing in ad hoc network", in Proc. ICC, 2005, pp. 3182 - 3186.

[29] C. Ma and Y. Yang, "Battery-aware routing in wireless ad hoc networks - part I: energy model", in Proc. $19^{\text {th }}$ ITC19, 2005, pp. $293-302$.

[30] X. Zheng, W. Guo, R. Liu, Y. Tian, "A new dynamic load-aware based load-balanced routing for ad hoc networks", in Proc. IEEE ICCCAS, 2004, pp. 407 - 411. 